\title{
Avaliação da Assistência Pré-Natal segundo o Modelo de Atenção às condições crônicas no Paraná
}

\author{
Evaluation of Prenatal Care according to the Care Model for chronic conditions in Paraná \\ Evaluación de la Atención Prenatal según el Modelo de Atención a condiciones crónicas en Paraná
}

Recebido: 03/03/2021 | Revisado: 10/03/2021 | Aceito: 11/03/2021 | Publicado: 19/03/2021

Laís Carolini Theis

ORCID: https://orcid.org/0000-0001-6346-8661 Universidade Positivo, Brasil

E-mail: laistheis@gmail.com

Karina Passero

ORCID: https://orcid.org/0000-0002-2010-2077 Universidade Regional de Blumenau, Brasil E-mail: karinapassero@gmail.com

Camila Michelle Westphal

ORCID: https://orcid.org/0000-0002-9390-6391 Universidade Regional de Bluemanu, Brasil E-mail: camilamichellew@gmail.com

Wanesa Aparecida da Costa

ORCID: https://orcid.org/0000-0001-9268-4080 Universidade Positivo, Brasil

E-mail: wanessabonadiman@gmail.com

Simone Tetu Moysés

ORCID: https://orcid.org/0000-0003-4861-9980 Pontifícia Universidade Católica do Paraná, Brasil E-mail: simone.moyses3@gmail.com

\begin{abstract}
Resumo
Avaliar a assistência pré-natal ofertada às mulheres atendidas no Sistema Único de Saúde do Paraná de modo a contribuir na tomada de decisão de gestores. Trata-se de um estudo transversal descritivo com abordagem quantitativa, realizado com gestantes que realizaram o acompanhamento pré-natal em serviços de saúde pública de uma região de saúde do estado do Paraná, no ano de 2018. Os dados foram coletados por meio de prontuário eletrônico e registros em planilhas dos gestores locais. Os resultados evidenciaram que das 89 gestantes que realizaram acompanhamento pré-natal, $84,3 \%$ foram captadas antes das 12 semanas, 55\% foram classificadas como risco habitual. Das gestantes com risco intermediário e alto, 73,7\% tiveram acesso ao serviço de atenção ambulatorial especializada. Entretanto, apenas 14,3\% das gestantes tiveram registro de idade gestacional em todas as consultas, $34,8 \%$ registro de altura uterina após 12 semanas, $37,1 \%$ registro de peso e $52,8 \%$ receberam o plano de cuidados realizados por profissionais de saúde. Apesar da maioria das mulheres serem classificada como risco habitual, $61,1 \%$ teve a cesárea como via de parto. Conclui-se que a assistência pré-natal tem sido ofertada de forma satisfatória. Entretanto, faz-se necessário que os gestores proporcionem capacitações assertivas aos profissionais envolvidos, para implementação adequada do Modelo de Atenção às Condições Crônicas visando desfechos positivos do período gravídico-puerperal.
\end{abstract}

Palavras-chave: Cuidado pré-natal; Qualidade da assistência à saúde; Saúde materna.

\begin{abstract}
Evaluate the prenatal care offered to women attended in the Unified Health System of Paraná in order to contribute to the decision making of managers. This is a descriptive cross-sectional study with a quantitative approach, carried out with pregnant women who underwent prenatal care. in public health services of a health region in the state of Paraná, in 2018. Data were collected through electronic medical records and records in spreadsheets of local managers. The results showed that of the 89 pregnant women who underwent prenatal care, $84.3 \%$ were captured before 12 weeks, $55 \%$ were classified as habitual risk. Of the pregnant women with intermediate and high risk, $73.7 \%$ had access to the specialized outpatient care service. However, only $14.3 \%$ of pregnant women had a record of gestational age in all consultations, $34.8 \%$ record a uterine height after 12 weeks, $37.1 \%$ record a weight and 52.8\% received the care plan performed by Health professionals. Despite the fact that most women are classified as habitual risk, $61.1 \%$ had cesarean section as the mode of delivery. It is concluded that prenatal care has been offered satisfactorily. However, it is necessary that managers provide assertive training to the professionals involved, for the proper implementation of the Care Model for Chronic Conditions aiming at positive outcomes of the pregnancy-puerperal period.
\end{abstract}


Keywords: Prenatal care; Quality of health care; Maternal health.

\section{Resumen}

Evaluar la atención prenatal que se brinda a las mujeres atendidas en el Sistema Único de Salud de Paraná con el fin de contribuir a la toma de decisiones de los gestores. Se trata de un estudio descriptivo transversal con enfoque cuantitativo, realizado con gestantes que se sometieron a atención prenatal. En público servicios de salud de una región de salud en el estado de Paraná, en 2018. Los datos fueron recolectados a través de historias clínicas electrónicas y registros en hojas de cálculo de los gerentes locales. Los resultados mostraron que de las 89 gestantes que se sometieron a atención prenatal, el 84,3\% fueron capturadas antes de las 12 semanas, el 55\% fueron clasificadas como de riesgo habitual. De las gestantes con riesgo intermedio y alto, el 73,7\% tuvo acceso al servicio de atención ambulatoria especializada. Sin embargo, solo el 14,3\% de las gestantes tuvo un registro de edad gestacional en todas las consultas, el 34,8\% registró una altura uterina a las 12 semanas, el 37,1\% registró un peso y el 52,8\% recibió el plan de atención realizado por los profesionales de la salud. A pesar de que la mayoría de las mujeres están clasificadas como de riesgo habitual, el $61,1 \%$ tuvo cesárea como modalidad de parto. Se concluye que la atención prenatal se ha brindado de manera satisfactoria. Sin embargo, es necesario que los gerentes brinden capacitación asertiva a los profesionales involucrados, para la adecuada implementación del Modelo de Atención a las Condiciones Crónicas con el objetivo de obtener resultados positivos del período embarazo-puerperal.

Palabras clave: Atención prenatal; Cualidad de la atención de salud; Salud materna.

\section{Introdução}

Inúmeros avanços foram conquistados desde a elaboração da Política Nacional de Atenção Integral à Saúde da Mulher, que contribuíram para as políticas de acesso e assistência pré-natal no país. Aliada às iniciativas de ampliação, qualificação e humanização da atenção à saúde da mulher, representam importante ferramenta para redução da mortalidade materna e infantil no país (Brasil, 2012a).

Entretanto, os indicadores de mortalidade materna ainda representam um desafio, considerando que de acordo com a Organização das Nações Unidas, um dos objetivos de desenvolvimento do milênio é a redução da mortalidade materna em três quintos (Vanderlei \& Frias, 2015). Cujo indicador, muito possivelmente, não será atingido, pois, o indicador se mantém elevado em todas as regiões do país (Silva et al., 2020).

A Rede Mãe Paranaense foi instituída no estado do Paraná com objetivo de prestar assistência materno-infantil, por meio da implementação de ações e medidas que favoreçam a redução dos indicadores de mortalidade materno-infantil, as quais precisam ser aplicadas através dos profissionais da saúde e da sociedade (Paraná, 2018).

O pré-natal é uma ferramenta indispensável que viabiliza o acompanhamento da evolução da gestação, cujas consultas são as melhores oportunidades para sanar as dúvidas da gestante e de sua parceria. O acompanhamento contínuo dos profissionais de saúde, por meio de consultas e exames promove o diagnóstico rápido dos fatores de risco associados à saúde da gestante e do bebê, e permite que a gestante exponha seus medos e anseios quanto ao progresso da gestação, o parto e o puerpério (Silva \& Andrade, 2020).

Sabe-se que existe relação entre a baixa adesão ao pré-natal e o aumento da probabilidade de desenvolvimento de complicações como parto prolongado, diabetes gestacional e hipertensão na gestação, e partindo dessa premissa acrescenta-se que existem práticas que devem, ou ao menos deveriam, ser executadas em todas as consultas do pré-natal, a exemplo da aferição da pressão arterial, da aferição do peso e verificação da altura uterina, além da realização dos exames preconizados como rotinas para cada trimestre gestacional, como ultrassonografias, hemograma, teste de tolerância oral à glicose, rastreio da sífilis, e vários outros que podem prevenir inúmeros agravos à saúde materna (Pereira et al., 2020).

Apesar disso, evidencia-se que o modelo de atenção à saúde do Brasil está em crise, a qual é exemplificada por um modelo de saúde que oferece uma terapêutica não holística e possui uma assistência majoritariamente voltada à demanda de quadros agudos e crônicos agudizados. Deste modo, o Modelo de Atenção às Condições Crônicas (MACC) é proposto como estratégia de enfrentamento e reorganização do modelo de atenção, para um cuidado contínuo e integrado em rede de atenção, a exemplo a Rede Mãe Paranaense. (Mendes, 2011). 
Neste contexto, o estado do Paraná implantou o MACC no âmbito das redes de atenção à saúde como proposta de cuidado e assistência materno-infantil por meio da Linha Guia da Rede Mãe Paranaense (Paraná, 2018).

Deste modo, o objetivo deste estudo é avaliar a assistência pré-natal ofertada às mulheres atendidas no Sistema Único de Saúde do Paraná. De modo a contribuir na tomada de decisão de gestores a respeito da implantação do Modelo de Atenção às Condições Crônicas.

\section{Metodologia}

Trata-se de uma pesquisa descritiva de delineamento transversal e abordagem quantitativa. $\mathrm{O}$ método quantitativo coleta dados por meio do uso de medições de grandezas e obtém-se por meio da metrologia, números com suas respectivas unidades. Este método permite com que sejam estabelecidas bases de dados que podem ser analisados por meio de técnicas matemáticas (Pereira et al., 2018).

O estudo foi realizado com gestantes que realizaram assistência e acompanhamento pré-natal em um município de pequeno porte da região noroeste do estado do Paraná, no período de janeiro a dezembro de 2018, cujo local foi pioneiro na implantação do Modelo de Atenção às Condições Crônicas no âmbito da Rede de Atenção Mãe Paranaense. Por tratar-se de um município de pequeno porte, com aproximadamente 3.672 habitantes (IBGE, 2020), optou-se por tornar sujeitos da pesquisa todas as mulheres gestantes do município.

Participaram do estudo 89 gestantes, sendo considerados critérios de inclusão: ser gestante, diagnosticada por profissional médico ou enfermeiro, faixa etária livre, estar cadastrada no serviço de atenção primária à saúde do município e residir no município. Como critérios de exclusão, gestantes com duplicidade de cadastro, gestantes que realizaram acompanhamento pré-natal na saúde suplementar ou privada.

A coleta de dados ocorreu por fontes secundárias, obtidos retrospectivamente por meio do sistema de informação de prontuário eletrônico, mediante autorização prévia para manipulação do banco de dados e aprovação pelo Comitê de Ética em Pesquisa Pontifícia Universidade Católica do Paraná, parecer nº 2.424.071/2017 e suas respectivas instituições coparticipantes. O estudo atendeu aos preceitos éticos estabelecidos na Resolução 466/2012 (Brasil, 2012b).

A coleta de dados incluiu variáveis para fins de caracterização da amostra (código do prontuário data de nascimento, número do cartão do SUS); variáveis associadas à Linha Guia da Rede Paranaense, objetivando conhecer a qualidade da assistência prestada (data de início do pré-natal, estratificação de risco, plano de cuidados, idade gestacional, altura uterina, peso, tipo de parto, aborto, acompanhamento da atenção ambulatorial especializada e desfechos).

Para gestantes acompanhadas na Atenção Ambulatorial Especializada, devido classificação de risco gestacional, os dados foram coletados de planilhas fornecidas por profissionais gestores do serviço.

A análise de dados foi realizada pelo software SPSS versão 23.0, por meio de análise estatística descritiva, frequências e medidas de tendência central (média).

O presente estudo seguiu os princípios e recomendações da iniciativa STROBE (Strengthening the Reporting of Observational Studies in Epidemiology), que se trata de uma lista de verificação que contém 22 itens com recomendações sobre o que deve ser incluído em uma descrição mais precisa e completa de estudos observacionais. Utilizou-se a versão traduzida e validada para o português (Malta et al., 2010).

\section{Resultados}

Participaram do estudo 89 gestantes, com média de idade de 26,7 anos, sendo idade mínima de 16 anos e máxima de 45 anos. A maioria, 75 (84,3\%) iniciou o acompanhamento pré-natal até doze semanas, conforme recomendação do Ministério da Saúde (Brasil, 2012a), e foram classificadas no primeiro trimestre como risco gestacional habitual, 49 (55\%), bem como no 
segundo trimestre, 51 (57,3\%), terceiro trimestre, 43 (48,3\%). A Tabela 1 descreve a estratificação de risco e o período de captação das gestantes para pré-natal.

Tabela 1. Estratificação do risco gestacional e vinculação pré-natal, Paraná, 2018.

\begin{tabular}{|c|c|c|c|c|c|c|}
\hline Variável & & & \multicolumn{2}{|l|}{$\mathbf{n}$} & \multicolumn{2}{|l|}{$\%$} \\
\hline \multicolumn{7}{|l|}{ Vinculação pré-natal } \\
\hline Entre 4 e 12 semanas & & & \multicolumn{2}{|l|}{75} & \multicolumn{2}{|c|}{84,3} \\
\hline Entre 13 e 26 semanas & & & \multicolumn{2}{|l|}{10} & \multicolumn{2}{|c|}{11,2} \\
\hline Entre 27 e 40 semanas & & & \multicolumn{2}{|l|}{3} & \multicolumn{2}{|c|}{3,4} \\
\hline Sem registro & & & \multicolumn{2}{|l|}{1} & \multicolumn{2}{|c|}{1,1} \\
\hline \multirow[t]{2}{*}{ Estratificação de risco } & $\mathbf{1}^{\mathrm{o}} \mathrm{t}$ & estre & \multicolumn{2}{|c|}{$2^{\circ}$ trimestre } & \multicolumn{2}{|c|}{$3^{\circ}$ Trimestre } \\
\hline & $\mathrm{n}$ & $\%$ & $\mathrm{n}$ & $\%$ & $\mathrm{n}$ & $\%$ \\
\hline Habitual & 49 & 55 & 51 & 57,3 & 43 & 48,3 \\
\hline Intermediário & 08 & 9 & 14 & 15,7 & 15 & 16,9 \\
\hline Alto & 11 & 12,4 & 14 & 15,7 & 19 & 21,3 \\
\hline Não classificada & 21 & 36,6 & 10 & 11,3 & 12 & 13,5 \\
\hline Total & 89 & 100 & 89 & 100 & 89 & 100 \\
\hline
\end{tabular}

Fonte: Dados da pesquisa (2018).

Com relação à assistência e o cuidado pré-natal apenas 3 (3,4\%) não tiveram o risco gestacional estratificado em nenhuma fase de acompanhamento, 47 (52,8\%) receberam plano de cuidados realizado pelos profissionais de saúde, 13 $(14,6 \%)$ tiveram registro de idade gestacional em todas as consultas durante todo o acompanhamento pré-natal, 31 (34,8\%) foi realizado mensuração de altura uterina em todas as consultas de pré-natal, a partir da 12a semana de gestação, e 33 (37,1\%) tiveram registro de peso em todas consultas de pré-natal, conforme descrito na Tabela 2. 
Tabela 2. Assistência pré-natal conforme Linha Guia Rede Mãe Paranaense, Paraná, 2018.

\section{Variável}

Plano de cuidados

Sim

Não $\mathrm{n}$

47

42

\section{$1^{\circ}$ trimestre}

$\mathrm{n}$

\section{Idade Gestacional}

Em todas as consultas

43

7

\section{$2^{\circ}$ trimestre}

n
48,3

28,1

5,6

$\%$
Parcialmente

Em nenhuma das

consultas

Não teve consulta

16

18

25

5

\section{Peso}

Em todas as consultas

Parcialmente

Em nenhuma das consultas

Não teve consulta

\section{Altura Uterina}

Em todas as consultas

12

Parcialmente

30

33,7

31

34,8

Em nenhuma das consultas

Não teve consulta
17

19,1

4,5

18

7
$\%$

52,8

47,2

\section{$3^{\circ}$ trimestre}

$\%$

n 
(100\%) a termo. Das gestantes acompanhadas, 2 (2,2\%) evoluíram para aborto espontâneo.

Entre as 19 gestantes que tiveram classificação de risco intermediário e alto no primeiro trimestre, 14 (73,7\%) realizaram pelo menos uma consulta de avaliação e acompanhamento no serviço de atenção ambulatorial especializado, com profissional obstetra. As gestantes encaminhadas para atenção especializada, 7 (50\%) deram continuidade da assistência prénatal no serviço especializado concomitante à assistência na atenção primária à saúde, 4 (28,6\%) receberam alta do serviço especializado, dando seguimento apenas na atenção primária.

\section{Discussão}

Os achados deste estudo indicam que a maioria das mulheres $(84,3 \%)$ iniciaram o acompanhamento do pré-natal até doze semanas de gestação, conforme preconizado pelo Ministério da Saúde (Brasil, 2012a) e pela Linha Guia da Rede Mãe Paranaense (Paraná, 2018). Outras pesquisas nacionais trazem resultados a respeito da captação precoce das gestantes. O estudo de Pereira et al. (2020), realizado com 6.545 gestantes cadastradas no SISPRENATAL e atendidas em equipes de Estratégia de Saúde da Família de Montes Claros - MG, no ano de 2016, revelou que a captação das gestantes no primeiro trimestre foi de 56,52\%. Ruiz et al. (2021) identificaram que a captação de mulheres até a $14^{a}$ semana de gestação foi de $81,6 \%$ em três Regionais de Saúde do Paraná. A pesquisa de Kinalski, Olivo e Giordani (2020), realizada com profissionais de ESFs de Santa Maria (RS), evidenciou que 69,2\% das mulheres realizaram a primeira consulta de pré-natal no primeiro trimestre gestacional.

Em comparação aos estudos supracitados, nossos achados a respeito da captação do pré-natal em até doze semanas se mostram satisfatórios. Entretanto, o fato de $15,7 \%$ das mulheres não ter sido vinculadas dentro deste prazo evidencia a necessidade de reavaliar as ações para captação das gestantes dentro do prazo recomendado, uma vez que a captação precoce (até a $12^{\mathrm{a}}$ semana de gestação) permite identificar possíveis fatores de risco e estabelecer cuidados ao pré-natal (Brasil, 2019), permitindo um acompanhamento de qualidade na assistência à saúde da mulher no ciclo gestacional.

A partir da estratificação de risco da gestante que é definida sua vinculação ao pré-natal e ao hospital, para que seja atendida conforme suas necessidades e no momento do parto (Paraná, 2018). Nossos resultados mostram que três mulheres não tiveram a estratificação de risco realizada em nenhuma fase do acompanhamento pré-natal, estando em discordância com o que a Linha Guia da Rede Mãe Paranaense propõe, ao indicar a importância da estratificação de risco na primeira e nas subsequentes consultas do pré-natal.

A pesquisa de Ruiz et al. (2021), também aponta um hiato na classificação de risco em quase metade da amostra. Outros problemas também foram observados quanto a esta prática, como no estudo de Amari et al. (2020), que apontou falhas nas estratificações de risco gestacionais, como erros na idade materna, presença de abortamento, entre outros. A não realização da estratificação de risco ou presença de erros pode prejudicar o acompanhamento pré-natal, pela impossibilidade de prever de forma assertiva em que ponto da rede de atenção a gestante se encontra, afetando seu vínculo aos hospitais de referência, e a garantia do parto com padrões de qualidade e segurança, objetivos estratégicos da Linha Guia da Rede Mãe Paranaense (Paraná, 2018).

Para as mulheres estratificadas, o risco habitual teve destaque em todos os trimestres gestacionais. Ainda assim, um número expressivo esteve classificado em risco intermediário e alto. Estas gestantes, por sua vez, receberam acompanhamento da atenção especializada. Embora a Unidade Básica deva ser sempre responsável pelo acompanhamento e seguimento das gestantes, a estratificação de risco permite que, quando necessário, estas sejam encaminhadas a serviços de maior complexidade, onde há oferta de procedimentos de maior densidade tecnológica e profissionais capacitados, a fim de oferecer assistência de qualidade no pré-natal e parto (Paraná, 2018; Brasil, 2012a). 
A gestante busca o serviço de referência no qual foi vinculada para o momento do parto. Os requisitos para que os hospitais e maternidades de risco habitual e risco intermediário façam parte da Rede Mãe Paraense, incluem, dentre outros, "possuir plano de ação para qualificação de atenção à gestante, tendo como objetivo a redução da taxa de cesárea para percentuais abaixo de 38\% do total de partos ocorridos no estabelecimento" (Paraná, 2018).

Apesar da mobilização através dos requisitos da Rede Mãe Paranaense e demais esforços do Ministério da Saúde para valorização do parto normal e redução do número de cesarianas, os resultados do presente estudo indicam que $61,1 \%$ das mulheres tiveram parto cesárea, ainda que a maioria delas foram estratificadas como baixo risco. No estudo de Barrios et al. (2021), realizado com mulheres atendidas em uma maternidade de risco habitual no Rio Grande do Sul, 31,9\% dos partos foram cesáreas. O estudo de Silva et al. (2020) aborda a utilização equivocada do parto cesáreo a nível nacional.

Que o avanço da obstetrícia trouxe melhoria para os indicadores de mortalidade materna e perinatal é inquestionável. Questiona-se seu uso indiscriminado, sem a real necessidade da intervenção cirúrgica, em que as desvantagens podem sobrepor as vantagens do procedimento (Brasil, 2017).

O plano de cuidados, apesar de proposto como estratégia de enfrentamento e reorganização do modelo de atenção e proposta de cuidado e assistência materno-infantil, foi utilizado por apenas 47 (52,8\%) dos casos (Mendes, 2011; Paraná, 2018). Deste modo, a utilização do plano ainda precisa ser difundida entre os profissionais e priorizada quando se trata de atenção à saúde materno-infantil.

O exame físico é um importante instrumento para acompanhar o desenvolvimento materno-infantil, devendo ser realizado em todas as consultas pré-natais. É composto pela avaliação de peso da gestante, idade gestacional, altura uterina, verificação da pressão arterial da mãe, avaliação dos batimentos cardiofetais e manobra de Leopold (Silva et al., 2021).

O acompanhamento da situação nutricional da gestante é considerado essencial durante as avaliações de pré-natal e é de responsabilidade da Equipe de Atenção Primária à Saúde (Paraná, 2018). O peso é um importante indicador para a avaliação do estado nutricional da mãe, e foi realizado na maior parte $(68,9 \%)$ das mulheres em todos os trimestres de gestação.

O cálculo da idade gestacional, assim como a data provável do parto, deve ser atualizado a cada atendimento. São informações que possibilitam o acompanhamento das ações de cuidado e que fornecem dados para criação de indicadores, e assim, gestão da condição de saúde (Brasil, 2019). Em nosso estudo, a idade gestacional foi avaliada em 70,7\% das mulheres durante todas as consultas.

Outro parâmetro que compõe o exame físico, é composto pela altura uterina, tal avaliação objetiva detectar restrição de crescimento intrauterino após a vigésima semana de gestação. Apesar de sua importância, os achados deste estudo evidenciaram que apenas $42,3 \%$ das mulheres tiveram altura uterina verificada em todas as consultas. Outros estudos também apontaram para a falta de registro deste parâmetro. O artigo de Camargos et al. (2020) identificou a ausência de registros no gráfico de altura uterina em $70,9 \%$ das gestantes avaliadas. Não foram avaliados os motivos pelos quais os profissionais não realizam a medição da altura uterina, ou se o fazem, porém não existe o registro no prontuário eletrônico. Desta forma, sugerem-se novas pesquisas relacionadas aos motivos pelo qual os profissionais deixam de registrar o dado em questão.

\section{Considerações Finais}

Este estudo permitiu avaliar a assistência pré-natal ofertada às mulheres atendidas no Sistema Único de Saúde do Paraná e evidenciar que a mesma tem sido executada dentro do contexto preconizado pelo Ministério da Saúde e da Linha Guia Rede Mãe Paranaense na região de saúde analisada. Foi perceptível que a procura e a captação ocorreram em tempo oportuno, antes de 12 semanas de gestação.

O seguimento do pré-natal foi satisfatório no que se refere à estratificação de risco, seguimento em serviço de atenção especializada nos casos classificados como risco intermediário e alto. Constatando-se desfechos positivos com relação ao 
nascimento em tempo oportuno (a termo), ausência de registros de mortes maternas e infantil e baixas taxas de aborto espontâneo.

No entanto, algumas lacunas com impacto negativo no cuidado foram identificadas, como o baixo registro ou mensuração da altura uterina, peso, idade gestacional, plano de cuidados e a escolha da via de parto ser em sua maioria cesárea, mesmo quando a maior parte das mulheres foi estratificada como baixo risco. Esses achados nos mostram riscos que interferem diretamente na assistência pré-natal e seus desfechos, sinalizando dessa forma a importância do olhar dos gestores para a educação permanente da equipe multiprofissional.

Este estudo contribui para que os gestores tenham um olhar assertivo para a (re) adequação aos protocolos, fluxos de atendimento e, ainda, de capacitações direcionadas às equipes de atuação na saúde materna, oferecendo dessa forma assistência pré-natal de qualidade. Recomendam-se novos estudos que busquem entender os determinantes das fragilidades e lacunas evidenciadas pelo diagnóstico realizado nesta pesquisa.

\section{Agradecimentos}

Agradecemos a Organização Pan Americana de Saúde ao financiamento para realização do estudo.

\section{Referências}

Amari, M. N., Carletto M. R., Borges, P. K. O., Amari, V.C. S., Fontes, A. L. G., Zielinski, J. N. P., \& Hilgemberg, A.(2020). Internações em unidade de terapia intensivan neonatal e a estratificação de risco gestacional do Programa Rede Mãe Paranaense. Braz. Journal of Develop 6(7), 42365-42377. $10.34117 / \mathrm{bjdv} 6 \mathrm{n} 7-012$.

Barrios, T. V., Souza, L. C. de, Diaz, C. M. G., Marques, C. T., Carrion, K. T. da S., Zamberlan, C., \& Menezes, F. L. (2021). Perfil de cesarianas de uma maternidade do interior do Rio Grande do Sul. Research, Society and Development, 10(1), e33610111747. 10.33448/rsd-v10i1.11747.

Brasil. Ministério da Saúde. (2012a). Atenção ao pré-natal de baixo risco - manual técnico. Brasília, DF.

Brasil. Ministério da Saúde. (2012b). Resolução $n^{\circ}$ 466, de 12 de dezembro de 2012. Diretrizes e normas regulamentadoras de pesquisas envolvendo seres humanos. Diário Oficial da União, Brasília, DF.

Brasil. Ministério da Saúde. (2017). Diretrizes Nacionais de Assistência ao Parto Normal. Brasília, DF.

Brasil. Ministério da Saúde. (2019). Saúde Da Mulher Na Gestação, Parto E Puerpério. São Paulo: Hospital Israelita Albert Einstein: Ministério da Saúde, 160.

Camargos, L. F.de, Lemos, P. L. Martins, E. F., \& Felisbino-Mendes, M. S. (2021). Avaliação da qualidade dos registros de cartões de pré-natal de mulheres urbanas. Escola Anna Nery, 25(1), e20200166.

Instituto Brasileiro de Geografia e Estatística (IBGE). (2020). Munhoz de Melo. Recuperado em: https://cidades.ibge.gov.br/brasil/pr/munhoz-demelo/panorama.

Kinalski, D. D. F., Olivo, V. M. F., \& Giordani, J. M. do A. (2020). Qualidade do pré-natal: impacto da infraestrutura e do processo de trabalho. Research, Society and Development, 9(9), 1-25. 10.33448/rsd-v9i9.7623.

Malta, M., Cardoso, L. O., Bastos, F. I., Magnanini, M. M., Silva, C. M. F. P. (2010). Iniciativa STROBE: subsídios para a comunicação de estudos observacionais. Rev. Saúde Pública, 44(3), 559-565. 10.1590/S0034-89102010000300021.

Mendes, E.V (2011). As redes de atenção à saúde. Brasília: OPAS.

Paraná. (2018). Linha Guia Rede Mãe Paranaense. Secretária de Estado Da Saúde Do Paraná, 1-63.

Pereira A. S., Shitsuka, D. M., Parreira, F. J., \& Shitsuka, R. (2018). Metodologia da pesquisa científica. UFSM. https://repositorio.ufsm.br/bitstream/handle/1/15824/Lic_Computacao_Metodologia-Pesquisa-Cientifica.pdf?sequence=1.

Pereira, J. S., Almeida E. W. S., Evangelista, C. B., Dias, C. L. O., Quadros, J. F. C., Oliveira, P. A. P., \& Dias, O. V. (2020). Perfil de gestantes atendidas no pré-natal em equipes de estratégia saúde da família. Saúde Coletiva (Barueri), 10(52), 2112-2123.

Ruiz, L. K. F. T., Franchi, J. V. de O., Colombo, N. C. R., Medeiros, F. F., Ferrari, R. A. P., Pelloso, S. M., \& Cardelli, A. A. M. (2021). Assistência pré-natal em serviços públicos de saúde do Estado do Paraná. Research, Society and Development, 10(2), e37010212543. 10.33448/rsd-v10i2.12543.

Silva, B. G. C. da, Lima, N.P., Silva, S. G. da, Antúnez, S. F., Seerig, L. M., Restrepo-Méndez, M. C., \& Wehrmeister, F. C. (2016). Mortalidade materna no Brasil no período de 2001 a 2012: tendência temporal e diferenças regionais. Revista Brasileira de Epidemiologia, 19(3), 484-493. 10.1590/19805497201600030002. 
Research, Society and Development, v. 10, n. 3, e36910313515, 2021

(CC BY 4.0) | ISSN 2525-3409 | DOI: http://dx.doi.org/10.33448/rsd-v10i3.13515

Silva, A. A. B. D., \& Andrade, C. (2020). O papel do enfermeiro na assistência, educação e promoção da saúde no pré-natal. Research, Society and Development, 9(10), e9989109477. 10.33448/rsd-v9i10.9477.

Silva, D.Z., Santos, M.G., Oliveira, S.C., Santos, F.H., \& Anjos, F.C.Q. (2020) Expoente sobre a prática de cesarianas no Brasil: análise a partir dos grupos de Robson. Revista de Patologia do Tocantins, 7(1): 105-107.

Silva, D. L. C. da, Cebriano, G. C.M., Figueiredo, H. R. P. P., Passos, I. C. G. A., Barbosa, K. L. R., Andrade, P. P. de, Santos, T.N.S., \& Santiago, V. E. L. (2021). Avaliação da qualidade e do entendimento das Gestantes e Puérperas na Atenção Primária no Pré-natal Research, Society and Development, 10(2), e20810212385. 10.33448/rsd-v10i2.12385.

Vanderlei, L. C. M. \& Frias, P. G. (2015). Avanços e desafios na saúde materna e infantil no Brasil. Rev. Bras. Saude Mater. Infant, 15(2), 157-158. $10.1590 / \mathrm{S} 1519-38292015000200001$. 